\title{
ASPECTOS BIOLÓGICOS DE ORIUS INSIDIOSUS (SAY, 1832) PREDANDO OVOS DE PLUTELLA XYLOSTELLA (L., 1758) E ANAGASTA KUEHNIELLA (ZELLER, 1879)
}

\section{J.P. Brito, A.M. Vacari, R.T. Thuler, S.A. De Bortoli}

UniversidadeEstadual Paulista, Faculdade deCiências Agráriase Veterinárias, Departamento de Fitossanidade, Laboratório de Biologia e Criação de Insetos, Via de Acesso Prof.Paulo Donato Castellane, s/no , CEP14884-900, Jaboticabal, SP, Brasil. E-mail. bortoli@fcav.unesp.br

\section{RESUMO}

O objetivo desse trabalho foi avaliar a biologia de Orius insidiosus alimentado com ovos de Plutella xylostella e Anagasta kuehniella. Os insetos foram provenientes da criação estoque do Laboratório de Biologia e Criação de Insetos do Departamento de Fitossanidade da FCAV/ UNESP. O experimento foi conduzido com ninfas de O. insidiosus, com idade entre 12-24h, uma por placa, num total de 50 ninfas (50 repetições). Em cada placa foram colocados, diariamente, ovos de $P$. xylostella ou ovos de A. kuehniella e um chumaço de algodão umedecido com água destilada. As avaliações foram realizadas diariamente. Os insetos que chegaram à fase adulta foram separados em casais, sendo colocados em placas. Foram avaliados os aspectos biológicos: duração, viabilidade e consumo dos estádios ninfais e da fase ninfal; longevidade de machos e fêmeas; consumo diário e total da fase adulta; ovos por dia; fecundidade das fêmeas; fertilidade dos ovos; período embrionário; períodos de pré-oviposição, oviposição e pós-oviposição. Além disso, também foram avaliados os parâmetros da tabela de vida de fertilidade. O predador $O$. insidiosus não apresenta diferenças significativas para suas características biológicas quando alimentado com as duas presas, porém melhores parâmetros de tabela de vida de fertilidade são observados quanto é alimentado com ovos de $P$. xylostella, sugerindo a sua possibilidade de utilização em criações massais desse inseto.

PALAVRAS-CHAVE: Biologia, controle biológico, percevejo predador.

\section{ABSTRACT}

BIOLOGICAL ASPECTS OF ORIUS INSIDIOSUS (SAY) FEEDING ON EGGS OF PLUTELLA XYLOSTELLA (LINNAEUS) AND ANAGASTA KUEHNIELLA (ZELLER). The objective of this work was to evaluate the biology of Orius insidiosus fed on eggs of Plutella xylostella and Anagasta kuehniella. The eggs used were obtained from the Laboratório de Biologia e Criação de Insetos, Departamento de Fitossanidade, FCAV/UNESP. The experiment was carried out with a total of 50 12-to-24-hou0r-old O. insidiosus nymphs, 1 per Petri dish (50 replications). P. xylostella or A. kuehniella eggs were places into each Petri dish daily, along with a small cotton pad moistened with distilled water. The evaluations were carried out daily. The adults were separated in couples, and placed in Petri dishes. The following biological aspects were evaluated: duration, survival rate and consumption of the nymph instars and of the nymph period; longevity of males and females; consumption per day and adult longevity; eggs per day; female fecundity; egg viability; embryonic period; preoviposition period, oviposition period, post-oviposition period. The fertility life table parameters were also evaluated. The predator $O$. insidiosus did not present significant differences for its biological characteristics, when feeding on P. xylostella and $A$. kuehniella eggs, however it showed improved fertility life table parameters when fedo $\mathrm{n}$ eggs of P. xylostella, suggesting the possibility of using these eggs in the mass rearing of this insect.

KEY WORDS: Biology, biological control, predator stinkbug.

\section{INTRODUÇÃO}

A produção de brássicas por meio de plantios sucessivos e a não eliminação dos restos culturais, principalmente para o repolho, juntamente com os fatores ambientais que favorecem o rápido crescimento das populações da traça-das-crucíferas, Plutella xylostella (Linnaeus, 1758)(Lepidoptera:Plutellidae), 
aumentam o potencial de dano e a multiplicação contínua da praga (BARRANTES; RODRIGUES, 1996; CASTELO BRANCO et al., 2003).

Com os prejuízos causados pela traça, a opção dos produtores de brássicas tem sido a aplicação intensiva de inseticidas, o que tem ocasionado a seleção de populações resistentes dessa praga a um grande número de inseticidas, como constatado de que aplicações em até três vezes semanais não reduziram os danos da traça (CASTELO BRANCO et al., 2001).

Apesar da utilização regular de inseticidas nas culturas das brássicas, muitos inimigos naturais são encontrados em meio à cultura, sendo que, quase em sua totalidade, os entomófagos constatados ou utilizados em experimentos e liberações massais são parasitoides de varios gêneros como Trichogramma, Oomyzus, Diadegma e Cotesia, principalmente (Cordero; CAVE, 1992; Ferreira et al., 2003; Thuler et al., 2007; Goulart et al., 2008). No entanto, pouco se sabe sobre a utilização de predadores para o controle da traça, à exceção de algumas respostas obtidas para espécies do gênero Chrysoperla (REDDY et al., 2004; MA et al., 2005).

Dentre os predadores presentes no agroecossistema das brássicas, os percevejos do gênero Orius têm sido frequentemente constatados em observações de campo, porém, pouco se sabe sobrea utilizaçãodesses insetos, seja em testes laboratoriais, seja em campo, apesar de sua simples presença na cultura já ser uma indicação da possibilidade de sua utilização como agente de controlebiológico de P.xylostella. A utilização de Orius insidiosus (Say, 1832) (Hemiptera: Anthocoridae) para controlar a traça-das-crucíferas pode ser uma alternativa para a redução das populações dessa praga, procurando-se minimizar os problemas ocasionados pelo uso indiscriminado deinseticidas.

Espécies de Orius são percevejos predadores generalistas que se alimentam de ampla faixa de presas, como tripes, ácaros, afídeos, ovos de lepidópteros e algumas lagartas pequenas (BUENO, 2000; Argolo et al., 2002). Mendes; Bueno (2001) observaram que Caliothrips phaseoli (Hood, 1912) (Thysanoptera: Thripidae) éuma presa adequada ao desenvolvimento e reprodução de $O$. insidiosus. OLIVEIRA et al. (2006) verificaram que a qualidade da presa tem alta relação com o alimento oferecido a ela, quando avaliaram a predação de Aphis gossipii (Glover, 1877) (Hemiptera: Aphididae), alimentados com diferentes cultivares de algodão.

O. insidiosus possui alta capacidade de busca e predação, além da habilidade de sobrevivência, mesmo em situações de ausência ou escassez de presas, podendo consumir fontes alimentares al- ternativas (BURGIO et al., 2004), o que pode facilitar sua permanência no campo nos períodos entre a colheita e a implantação de novos plantios de brássicas. Tendo em vista a possibilidade de utilização desse predador na cultura em questão, este trabalho teve como objetivo avaliar a biologia de $O$. insidiosus alimentado com ovos de P. xylostella, durante seu desenvolvimento e a adequação do alimento do predador por meio da obtenção da tabela de vida de fertilidade.

\section{MATERIALEMÉTODOS}

O experimento foi conduzido em laboratórioa 25 $\pm 1^{\circ} \mathrm{C}$ de temperatura, $70 \pm 10 \%$ de umidade relativa e fotofase de $12 \mathrm{~h}$. Oexperimento foi conduzido colocando-se uma ninfa de O. insidiosus, com idade entre $12-24 \mathrm{~h}$, por placa de Petri $(6 \mathrm{~cm}$ de diâmetro por $2 \mathrm{~cm}$ de altura), oriundas da criação mantida no laboratório. Foram realizados dois tratamentos. O primeiro, considerado testemunha, foi composto por ninfas alimentadas diariamente com ovos inviabilizados do piralídeo Anagasta kuehniella (Zeller, 1879) e o segundo por ninfas alimentadas também diariamente com ovos de P. xylostella, oriundos da criação de manutenção. Em ambos os casos os predadores receberam ovos "ad libitum". Ao total foram utilizadas 50 ninfas por tratamento que formaram 50 repetições. A umidade no interior das placas foi mantida colocando-se um chumaço de algodão de $1 \mathrm{~cm}^{2}$ umedecido com água destilada. Essas unidades foram posteriormente vedadas para evitar a fuga do inseto.

As avaliações relativas às mudanças de ínstar e à predação foram realizadas diariamente empregando-se um microscópio estereoscópico. Os insetos que chegaram à fase adulta foram separados por sexo pela observação da genitália e os casais formados foram colocados em placas de Petri $(6 \mathrm{~cm}$ de diâmetro por $2 \mathrm{~cm}$ de altura). Em ambos os tratamentos foi colocada diariamente, para a oviposição, uma haste de picão-preto (Bidens pilosa L.) por placa, até a morte dos adultos. A manutenção da umidade foi realizada colocando-se um chumaço algodão umedecido com água destilada. O número de posturas foi avaliado após $24 \mathrm{~h}$ e, posteriormente, as hastes foram colocadas nas placas até a eclosão das ninfas.

Os parâmetros avaliados foram: período, viabilidade e consumo diário de cada estádio ninfal e total, longevidade, número deovos / dia, fecundidade, fertilidade, período embrionário, pré-oviposição, oviposição e pós-oviposição.

Com os dados biológicos obtidos foram determinados os parâmetros necessários para a cons- 
trução da tabela de vida de fertilidade, segundo Birch (1948), Silveira Neto et al. (1976), SOUtHWOOD (1978) e PRICE (1984), onde: $x$ = ponto médio de cada idade das fêmeas parentais, idade essa considerada desde a fase de ovo; $1 x$ = expectativa de vida até a idade $x$, expressa como uma fração de uma fêmea; $m x$ = fertilidade específica ou número de descendentes por fêmea produzidos na idade $x$ e que originarão fêmeas; lx.mx = número total de fêmeas nascidas na idade $x$. Utilizando-se os parâmetros de crescimento resultantes da tabela de vida, foram calculados os valores de $\mathrm{R}_{\mathrm{o}}=$ taxa líquida, ou seja, a taxa de aumento populacional, considerando fêmeas de uma geração para outra, ou ainda, o número de fêmeas geradas por fêmea parental por geração; $\mathrm{T}=$ tempo médio de geração ou duração média de uma geração; $r_{m}=$ capacidade inata de aumentar em número ou taxa intrínseca de aumento; $\lambda=$ razão finita de aumento, definida como o número de vezes que a população multiplica em uma unidade de tempo. Além desses parâmetros, foi também determinado o TD = tempo necessário para a população duplicar em número, segundo KREBS (1994).

Os dados foram submetidos à análise de variância, com comparação das médias pelo teste de Tukey $(\mathrm{P}<0,05)$.

\section{RESULTADOSEDISCUSSÃO}

O desenvolvimento ninfal de O. insidiosus não foi alterado durante os três primeiros ínstares, independentemente da presa utilizada. No entanto, a duração do quarto estádio ninfal foi maior para ninfas que se alimentaram de ovos de A. kuehniella $(3,21$ dias) diferindo das que predaram ovos de P.xylostella (2,33 dias). No quinto estádio ninfal houve uma inversão nesses resultados e as ninfas que se alimentaram de ovos de $P$. xylostella tiveram tempo de desenvolvimento maior que sob alimentação com ovos de A. kuehniella. Apesar dessa constatação e analisando-se conjuntamente a duração de todos os estádios, nota-se uma redução no período ninfal total, quando o alimento foi ovos de P. xylostella (14,92 dias), em comparação a ovos de A. kuehniella (15,55 dias) (Tabela 1$)$.

Os dados encontrados aproximaram-se daqueles obtidos por Mendes et al. (2005), os quais evidenciam que o tipo de alimento é um dos principais fatores influenciadores no desenvolvimento ninfal de O. insidiosus, chegando a alterar o seu comportamento em condições de campo. Constataram também que o período ninfal de O. insidiosus alimentado com ovos de A. kuehniella, A. gossypii e C. phaseoli foi de 13,1; 11,2 e 10,2 dias, respectiva- mente. Observando ainda que a alimentação com ovos de A. kuehniella causou uma diferenciação no período de desenvolvimento das ninfas que originaram fêmeas (12,1 dias), em relação àquelas que originaram machos (12,4 dias).

As viabilidades das ninfas nos primeiros quatro ínstares variaram entre 92 e 100\%, sem diferenciação significativa, sendo observada diferença apenas no quinto estádio, com uma viabilidade maior para ninfas que sealimentaram deovos de A. kuehniella. Noentanto, a viabilidade total não sofreu influência entre os ínstares, constatando-se uma diferença significativa entre os tratamentos de $12 \%$ (Tabela 1). Esses resultados assemelharam-se àqueles de MENDES et al. (2005) cujas viabilidades variaram de 83 e $96 \%$, nos cinco ínstares de ninfas alimentadas com ovos de $A$. kuehniella.

Como foram oferecidos ovos de P. xylostella e $A$. kuehniella "adlibitum", foi observado também o consumodeovos pelas ninfas. Assim, o consumo aumentou à medida que as ninfas mudaram de estádio. Durante a fase ninfal foram consumidos 1,$39 ; 3,20 ; 3,74 ; 6,37$; 8,64 e 86,99 ovos de $P$. xylostella e 1,$65 ; 3,27 ; 4,75 ; 8,08$; 8,05 e 95,37 de A. kuehniella para o primeiro, segundo, terceiro, quarto, quinto e período ninfal total, respectivamente; sendo que as diferenças significativas no consumo, à exceção do quinto ínstar, indicaram sempreum maior consumo de ovos de A. kuehniella (Tabela 1). Valores semelhantes foram observados por CARvALHOetal. (2005) que verificaram consumo de 2,5;3,8; 7,2; 10,6 e 15,3 ovos de A. kuehniella para o primeiro, segundo, terceiro, quarto e quinto estádios de ninfas do antocorídeo Orius thyestes (Herring, 1966), respectivamente.

Também não foram observadas diferenças significativas para a longevidade demachos edefêmeas de O. insidiosus que se alimentaram de ovos das duas presas.

O consumo diário de ovos de P. xylostella $(5,90)$ e A. kuehniella $(6,05)$ por adultos de O. insidiosus foi semelhante, assim como o consumo total durante toda a fase adulta (Tabela 2). Esses resultados foram semelhantes aos observados por GUEDES (2006) que verificou que fêmeas de $O$. insidiosus predando ovos de P. xylostella consumiram 6,43 ovos diariamente.

As fêmeas alimentadas com ovos de P.xylostella e A. kuehniella tiveram desempenho reprodutivo semelhante, não sendo observada diferença significativa entre os números de ovos por dia, fecundidade, viabilidade dos ovos e período embrionário (Tabela 3). Em comparação com fêmeas de $O$. thyestes alimentadas com ovos de A. kuehniella apresentaram menor consumo de ovos por dia e ovos por fêmea, sendo 5,9 ovos / dia e 52,8 ovos / fêmea (CARvALHO et al., 2006). 


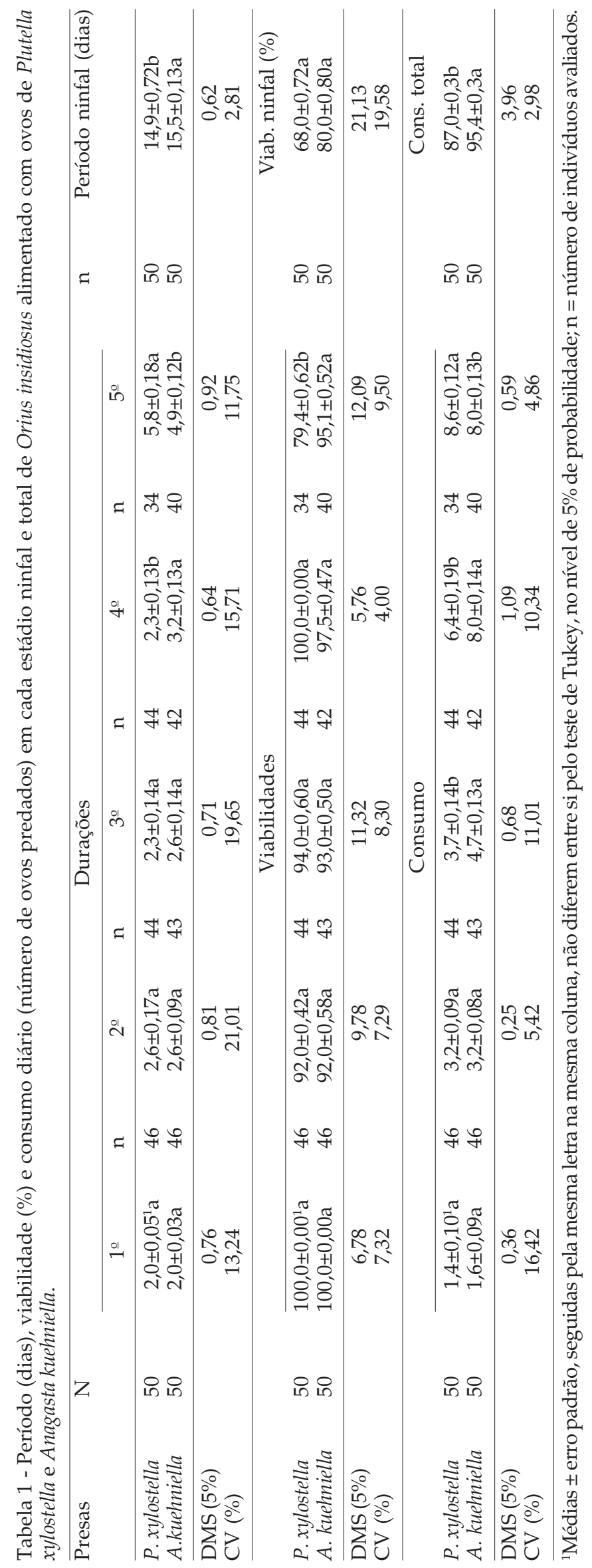


Tabela 2 - Longevidade de machos e fêmeas, consumo diário e total médio de adultos de Orius insidiosus alimentados com ovos de Plutella xylostella e Anagasta kuehniella.

\begin{tabular}{|c|c|c|c|c|c|c|c|c|}
\hline Tratamentos & $\mathrm{n}$ & $\begin{array}{l}\text { Long. machos } \\
\text { (dias) }\end{array}$ & $\mathrm{n}$ & $\begin{array}{l}\text { Long. fêmeas } \\
\text { (dias) }\end{array}$ & $\mathrm{n}$ & $\begin{array}{c}\text { Consumo } \\
\text { diário }\end{array}$ & $\mathrm{N}$ & $\begin{array}{c}\text { Consumo } \\
\text { total }\end{array}$ \\
\hline Plutella xylostella & 15 & $7,00 \pm 0,16^{1}$ & 14 & $6,78 \pm 0,29$ & 29 & $5,90 \pm 0,11$ & 29 & $35,92 \pm 0,95$ \\
\hline Anagasta kuehniella & 16 & $6,47 \pm 0,26$ & 16 & $6,36 \pm 0,29$ & 32 & $6,05 \pm 0,18$ & 32 & $28,25 \pm 1,02$ \\
\hline DMS (5\%) & & 1,86 & & 2,14 & & 0,85 & & 13,25 \\
\hline CV (\%) & & 36,99 & & 31,95 & & 17,58 & & 28,79 \\
\hline $\mathrm{F}$ & & $0,34^{\mathrm{ns}}$ & & $0,17^{\mathrm{ns}}$ & & $0,12^{\mathrm{ns}}$ & & $1,44^{\mathrm{ns}}$ \\
\hline
\end{tabular}

${ }^{1}$ Médias \pm erro padrão, seguidas pela mesma letra na mesma coluna, não diferem entre si pelo teste de Tukey, ao nível de $5 \%$ de probabilidade; $n=$ número de indivíduos avaliados.

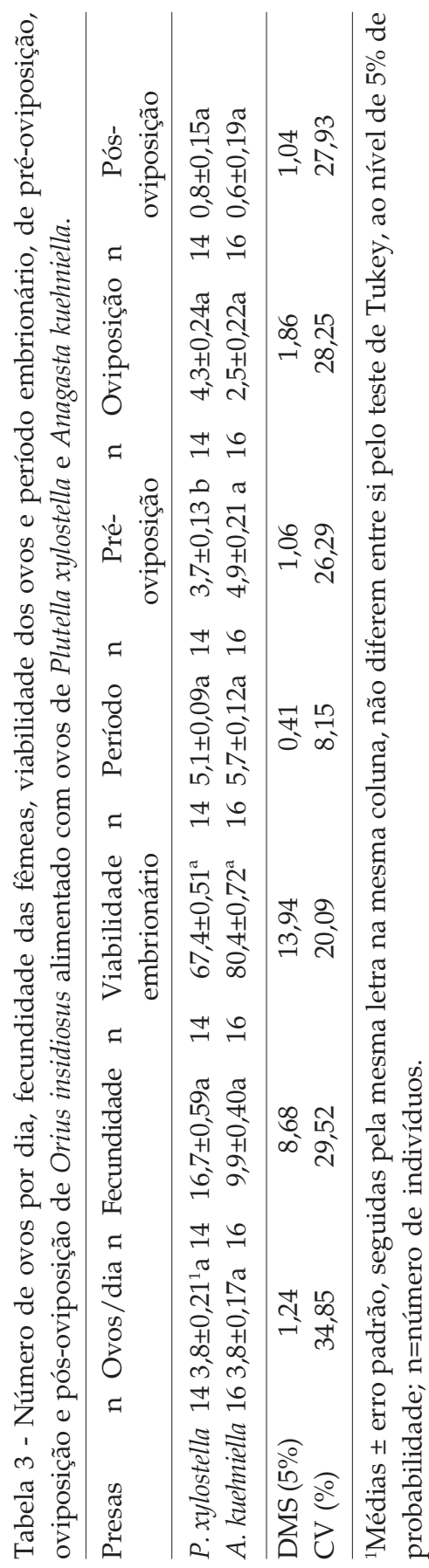

Argolo et al. (2002) também verificaram maior consumo com fêmeas de O. insidiosus para o número de ovos por fêmea, correspondendo a 195,2 ovos, porém o número de ovos por dia foi semelhante, 3,4 ovos. Essas diferenças possivelmente podem estar relacionadas à longevidade, sendo maior do que a observada na presente pesquisa.

O período de pré-oviposição foi maior para fêmeas que se alimentaram de ovos de A. kuehniella (4,90 dias) em comparação àquelas onde se utilizou como alimento ovos de $P$. xylostella $(3,70$ dias), observando-se uma redução de 1,2 dias nessa fase, em função das presas empregadas (Tabela 2).

Os períodos de oviposição e pós-oviposição não foram influenciados pelo alimento utilizado pelas fêmeas dos percevejos predadores (Tabela 3). ARGOLO et al. (2002) obtiveram respostas semelhantes, verificando que aquelas de $O$. insidiosus alimentadas com ovos de A. kuehniella têm período de pré-oviposição de 3,3 dias, porém foi constatado maior período de oviposição, 44,4 dias.

Alguns parâmetros da tabela de vida de $O$. insidiosus foram afetados pela alimentação do predador (Tabela 4). A taxa líquida de reprodução $\left(R_{\mathrm{o}}\right)$, apesar da diferença numérica, foi semelhante para os dois tratamentos, sendo 6,61 para P. xylostella e 2,40 para A. kuehniella. Para o tempo médio de geração $(\mathrm{T})$ ocorreu o oposto, havendo diferença significativa entre o menor valor, encontrado no tratamento com $P$. xylostella (24,96 dias), e o maior com A. kuehniella (27,26 dias). A taxa intrínseca de crescimento populacional $\left(\mathrm{r}_{\mathrm{m}}\right)$ e a razão finita de crescimento populacional $(\lambda)$ foram significativamente maiores para o tratamento $P$. xylostella, enquanto o tempo necessário para a população duplicar em número (TD) foi significativamente menor quando o predador foi alimentado com ovos dessa presa. 
Tabela 4 - Resultado da tabela de vida de fertilidade de Orius insidiosus alimentado com ovos de Plutella xylostella e Anagasta kuehniella.

\begin{tabular}{lccccr}
\hline Tratamentos & $\mathrm{R}_{0}$ & $\mathrm{~T}$ & $\mathrm{r}_{\mathrm{m}}$ & $\lambda$ & \multicolumn{1}{c}{$\mathrm{TD}$} \\
\hline Plutella xylostella & $6,61 \pm 0,40^{1}$ & $24,96 \pm 0,14 \mathrm{~b}$ & $0,0679 \pm 0,0336 \mathrm{a}$ & $1,0706 \pm 0,0347 \mathrm{a}$ & $7,94 \pm 0,50 \mathrm{~b}$ \\
Anagasta kuehniella & $2,40 \pm 0,21$ & $27,26 \pm 0,21 \mathrm{a}$ & $0,0288 \pm 0,0273 \mathrm{~b}$ & $1,0293 \pm 0,0276 \mathrm{~b}$ & $16,53 \pm 1,58 \mathrm{a}$ \\
\hline DMS (5\%) & 4,31 & 1,26 & 0,0348 & 0,0367 & 2,22 \\
CV $(\%)$ & 25,58 & 3,32 & 29,43 & 2,40 & 7,99 \\
F & $5,07^{\text {ns }}$ & $17,67^{* *}$ & $6,70^{*}$ & $6,71^{*}$ & $25,99^{* *}$ \\
\hline
\end{tabular}

${ }^{1}$ Médias \pm erro padrão, seguidas pela mesma letra na mesma coluna, não diferem entre si pelo teste de Tukey, ao nível de $5 \%$ de probabilidade

$\mathrm{R}_{\mathrm{o}}=$ Taxa líquida de reprodução (fêmea/fêmea)

$\mathrm{T}=$ Tempo médio de geração (dias)

$\mathrm{r}_{\mathrm{m}}=$ Taxa intrínseca de crescimento populacional

$\lambda=$ Taxa finita de crescimento populacional (fêmeas/dia)

$\mathrm{TD}=$ Tempo necessário para a população duplicar em número

\section{CONCLUSÃO}

Ovos de P. xylostella foi a presa que permitiu melhor desenvolvimento de $O$. insidiosus, em relação a ovos de A. kuehniella, sugerindo a possibilidade de uso desse predador no controle da traça-dascrucíferas, ou mesmo a utilização de ovos dessa praga como fonte alimentar em criações massais do inimigo natural, quando sua obtenção for mais fácil ou menos onerosa que a da presa alternativa.

\section{REFERÊNCIAS}

ARGOLO, V.M.; BUENO, V.H.P.; SILVEIRA, L.C.P. Influência do fotoperíodo na reprodução e longevidade de Orius insidiosus (Say) (Heteroptera: Anthocoridae). Neotropical Entomology, v.31, p.257-261, 2002.

BARRANTES, A.J.A.; RODRIGUEZ, V.C.L. Abundancia estacional y dano de Plutella xylostella (L.) (Lepidoptera: Plutellidae) y el cultivo de repollo, durante la epoca seca en Alfaro Ruiz, Alajuela, Costa Rica. Manejo Integrado de Plagas, v.39, p.17-24, 1996.

BIRCH, L.C. The entrinsic rate of natural increase of on insect population. Journal of Animal Ecolology, v.17, p.1526, 1948.

BUENO, V.H.P. Desenvolvimento e multiplicação de percevejos predadores do gênero Orius Wolff. In: BUENO, V.H.P. (Ed.). Controle biológico de pragas: produção massal e controle de qualidade. Lavras: UFLA, 2000. p. 69-90.

BURGIO, G.; TOMMASINI, M.G.; VAN LENTEREN, J.C. Population dynamics of Orius laevigatus and Frankliniella occidentalis: a mathematical modeling approach. Bulletin of Insectology, v.57, p.131-135, 2004.
CASTELO BRANCO, M.; FRANÇA, F.H.; MEDEIROS, M.A.; LEAL, J.G.T. Uso de inseticidas para o controle da traça-do-tomateiro e traça-das-crucíferas: um estudo de caso. Horticultura Brasileira, v.19, p.60-63, 2001.

CASTELO BRANCO, M.; FRANÇA, F.H.; PONTES, L.A.; AMARAL, P.S.T. Avaliação da suscetibilidade a inseticidas em populações de traça-das-crucíferas de algumas áreas do Brasil. Horticultura Brasileira, v.21, p.549-552, 2003.

CARVALHO, L.M.; BUENO, V.H.P.; MENDES, S.M. Desenvolvimento, consumo ninfal e exigências térmicas de Orius thyestes Herring (Hemiptera: Anthocoridae). Neotropical Entomology, v.34, p.607-612, 2005.

CARVALHO, A.R.; BUENO, V.H.P.; PEDROSO, E.C.; KON, L.I.; DINIZ, A.J.F.; SILVA, R.J. Influence of photoperiod on Orius thyestes Herring (Hemiptera: Anthocoridae) reproduction and longevity. Neotropical Entomology, v.35, p.489-492, 2006.

CORDERO, J.; CAVE, R.D. Natural enemies of Plutella xylostella (L.) (Lepidoptera: Plutellidae) on crucifers in Honduras. Entomophaga, v.37, p.397-407, 1992.

FERREIRA, S. W. J; BARROS, R.; TORRES, J. B. Exigências térmicas e estimativa do número de gerações de Oomyzus sokolowskii (Kurdjumov) (Hymenoptera: Eulophidae), para regiões produtoras de crucíferas em Pernambuco. Neotropical Entomology, v.32, p.407-411, 2003.

GOULART, R.M.; DE BORTOLI, S.A.; THULER, R.T.; PRATISSOLI, D.; VIANA, C.L.T.P.; VOLPE, H.X.L.

Avaliação da seletividade de inseticidas a Trichogramma ssp. (Hymenoptera: Trichogrammatidae) em diferentes hospedeiros. Arquivos do Instituto Biológico, São Paulo, v.75, n.1, p.69-77, 2008. 
GUEDES, I.V. Resposta funcional e numérica do predador Orius insidiosus (Say., 1832) (Hemiptera: Anthocoridae) com diferentes presas. 2006. 69f. Dissertação (Mestrado em Entomologia Agrícola) - Universidade Estadual Paulista, Faculdade de Ciências Agrárias e Veterinárias, Jaboticabal, 2006.

KREBS, C.J. Ecology: The experimental analysis of distribution and abundance. New York: Harper \& Row, 1994. 801p.

MA, J.; LI, Y.Z.; KELLER, M.; REN, S.X. Functional response and predation of Nabis kinbergii (Hemiptera: Nabidae) to Plutella xylostella (Lepidoptera: Plutellidae). Insect Science, v.12, p.281-286, 2005.

MENDES, S.M.; BUENO, V.H.P. Biologia de Orius insidiosus (Say) (Hemiptera: Anthocoridae) alimentado com Caliothrips phaseoli Hood (Thysanoptera: Thripidae). Neotropical Entomology, v.30, p.423-428, 2001.

MENDES, S.M.; BUENO, V.H.P.; CARVALHO, L.M. Desenvolvimento e exigências térmicas de Orius insidiosus (Say) (Hemiptera: Anthocoridae). Revista Brasileira de Entomologia, v.49, p.575-597, 2005.

OLIVEIRA, J.E.M.; DE BORTOLI, S.A.; GUEDES, I.V . Resposta funcional de Orius insidiosus (Say, 1832) a diferentes densidades de Aphis gossypii (Glover, 1877). Revista de Biologia e Ciência da Terra, v.6, p.63-72, 2006.

PRICE, P.W. Insect ecology. New York: John Willey, 1984. 607p.

REDDY, G.V.P.; TABONE, E.; SMITH, M.T. Mediation of host selection and oviposition behavior in the diamondback moth Plutella xylostella and its predator Chrysoperla carnea by chemical cues from cole crops. Biological control, v.29, p.270-277, 2004.

SILVEIRA NETO, S.; NAKANO, O.; BALDIN, D.; VILLANOVA, N.A. Manual de ecologia dos insetos. São Paulo: Agronômica Ceres, 1976. 419p.

SOUTHWOOD, T.R.E. Ecological methods. London: Chapman and Hall, 1978. 524p.

THULER, R.T.; VOLPE, H.X.L.; DE BORTOLI, S.A.; GOULART, R.M.; VIANA, C.L.T.P. Metodologia para avaliação da preferência hospedeira de parasitóides do gênero Trichogramma Westood. Boletin de Sanidad Vegetal. Plagas, v.33, p.333-340, 2007.

Recebido em 9/2/09

Aceito em 5/10/09 